\title{
An open label randomized Phase III trial of nivolumab or nivolumab plus ipilimumab vs platinum doublet chemotherapy (PT-DC) in patients with chemotherapy-naive stage IV or recurrent non-small cell lung cancer (NSCLC) (CheckMate 227)
}

\author{
Matthew Hellmann ${ }^{1 *}$, Suresh Ramalingam², Martin Reck ${ }^{3}$ Ken O'Byrne ${ }^{4}$, Luis Paz-Ares ${ }^{5}$, Christopher T Harbison ${ }^{6}$, \\ Prabhu Bhagavatheeswaran ${ }^{6}$, Faith Nathan ${ }^{6}$, Julie Brahmer ${ }^{7}$
}

From 30th Annual Meeting and Associated Programs of the Society for Immunotherapy of Cancer (SITC 2015) National Harbor, MD, USA. 4-8 November 2015

\section{Background}

Patients with advanced NSCLC are treated with first-line PT-DC, which is associated with a median OS of $8-10$ months and 1-year and 2-year survival rates of 30-40\% and $10-15 \%$, respectively. Nivolumab (a fully human IgG4 anti-programmed death-1 immune checkpoint inhibitor antibody) alone and in combination with ipilimumab (a fully human IgG4 cytotoxic T-lymphocyte antigen-4 immune checkpoint inhibitor antibody) has demonstrated encouraging clinical benefit across multiple tumor types. Two randomized Phase III trials demonstrated superior survival with nivolumab vs docetaxel in previously-treated patients with advanced squamous (SQ) (CheckMate 017) and non-squamous (non-SQ) NSCLC (CheckMate 057). Preliminary results of a Phase I study (CheckMate 012) of nivolumab with or without ipilimumab demonstrate acceptable safety and encouraging activity in first-line metastatic NSCLC across histologies. This Phase III trial (CheckMate 227) evaluates nivolumab monotherapy and nivolumab plus ipilimumab combination regimens vs PT-DC in patients with chemotherapy-naïve stage IV or recurrent SQ and non-SQ NSCLC.

\begin{abstract}
Methods
Adult patients with stage IV or recurrent NSCLC, ECOG performance status $\leq 1$, no prior systemic anticancer therapy, and measureable disease per RECIST version 1.1 are eligible. Tissue will be evaluated for programmed death-ligand 1 (PD-L1) expression during screening. Patients who have untreated CNS metastases are ineligible. Patients will be randomized to nivolumab monotherapy, nivolumab plus ipilimumab combination regimens, or PT-DC. PT-DC will be administered according to histology (gemcitabine with cisplatin or carboplatin for SQ and pemetrexed with cisplatin or carboplatin for non-SQ). Patients will receive treatment until progression or unacceptable toxicity. The co-primary endpoints are overall survival and progression-free survival in patients receiving nivolumab monotherapy or nivolumab plus ipilimumab combination regimens vs patients receiving PT-DC. Secondary endpoints include objective response rate in nivolumab monotherapy or nivolumab plus ipilimumab combination regimens vs PT-DC and disease related symptom improvement measured by the Lung Cancer Symptom Scale in all patients.
\end{abstract}

\section{Trial registration}

ClinicalTrials.gov identifier NCT02477826.

Memorial Sloan Kettering Cancer Center, New York, NY, USA

Full list of author information is available at the end of the article 


\section{Authors' details}

${ }^{1}$ Memorial Sloan Kettering Cancer Center, New York, NY, USA. ${ }^{2}$ Winship Cancer Institute, Emory University, Atlanta, GA, USA. ${ }^{3}$ Krankenhaus Grosshansdorf Gmbh, Ahrensburg, Germany. ${ }^{4}$ Cancer Services, Princess Alexandra Hospital, Brisbane, QLD, Australia. ${ }^{5}$ Hospital Universitario Virgen del Rocio, Sevilla, Spain. ${ }^{6}$ Bristol-Myers Squibb, Princeton, NJ, USA. ${ }^{7}$ Sidney Kimmel Comprehensive Cancer Center at Johns Hopkins, Baltimore, MD, USA.

Published: 4 November 2015

doi:10.1186/2051-1426-3-S2-P154

Cite this article as: Hellmann et al: An open label randomized Phase III trial of nivolumab or nivolumab plus ipilimumab vs platinum doublet chemotherapy (PT-DC) in patients with chemotherapy-naïve stage IV or recurrent non-small cell lung cancer (NSCLC) (CheckMate 227). Journal for ImmunoTherapy of Cancer 2015 3(Suppl 2):P154.

Submit your next manuscript to BioMed Central and take full advantage of:

- Convenient online submission

- Thorough peer review

- No space constraints or color figure charges

- Immediate publication on acceptance

- Inclusion in PubMed, CAS, Scopus and Google Scholar

- Research which is freely available for redistribution

Submit your manuscript at www.biomedcentral.com/submit
C Biomed Central 